\title{
Time Variant Change Analysis in Satellite Images
}

\author{
Rachita Sharma \\ Computer Science \& Engg. Deptt. \\ GNIT Girls Institute of Technology \\ Greater Noida, India
}

\author{
Sanjay Kumar Dubey \\ Assistant Professor, Computer Science \& Engg. Deptt. \\ Amity University, Uttar Pradesh \\ Sec. 125, Noida, India
}

\begin{abstract}
This paper describes the time variant changes in satellite images using Self Organizing Feature Map (SOFM) technique associated with Artificial Neural Network. In this paper, we take a satellite image and find the time variant changes using above technique with the help of MATLAB. This paper reviews remotely sensed data analysis with neural networks. First, we present an overview of the main concepts underlying Artificial Neural Networks (ANNs), including the main architectures and learning algorithms. Then, the main tasks that involve ANNs in remote sensing are described. We first make a brief introduction to models of networks, for then describing in general terms Artificial Neural Networks (ANNs). As an application, we explain the back propagation algorithm, since it is widely used and many other algorithms are derived from it. There are two techniques that are used for classification in pattern recognition such as Supervised Classification and Unsupervised Classification. In supervised learning technique the network knows about the target and it has to change accordingly to get the desired output corresponding to the presented input sample data. Most of the previous work has already been done on supervised classification. In this study we are going to present the classification of satellite images using unsupervised classification method of ANN.
\end{abstract}

Keywords-Satellite Images; SOFM; ANN; Supervised Classification, Unsupervised classification.

\section{INTRODUCTION}

Time series is a chain of observations that take part according to the time like weekly series of newspaper, hourly observations of news happening in the city, number of criminal records etc. Most popular fields of time variant changes are business economics, remote sensing and weather forecasting [1]. Basically time variant changes are dependent on some current adjacent observations. In time variant changes the goal is to create a prototype that can reveal the current process and predict the future of the measured process by calculating the values of certain variables sequentially in time. Usually data is incomplete and also having noise. So we try to find exact data possible be compensated by adjustment of the input series value.

There are two statistical methods to gain temporal information of an image. First is linear i.e. AR and ARMA and second is nonlinear i.e. NARMAX and MARS [2]. In now a day neural network in time variant prediction is convert temporal sequence into concatenated vector via a tapped delay line and to feed the resulting vector as input to a network. Most recurrent neural networks are trained via supervised learning rules. However in temporal sequence analysis unsupervised network could reveal useful information from the temporal sequence at hand in analogy to unsupervised neural networks reported power in cluster analysis and dimensionality reduction [3]. In unsupervised learning or self organizing learning provision is made for task independent measure of the quality of representation that the network is required to learn, and free parameters of the network are optimized with respect to that measure, once the network has become tuned to form internal representation for encoding features of the input and thereby to create new classes automatically [4].

Artificial Neural Networks are the computing models that are inspired by biological neural network and provide new directions to solve problems arising in natural tasks. In particular, it is hoped that neural network would extract the relevant features from the input data and perform a pattern recognition task by learning from examples without explicitly stating the rules for performing the task.

Currently most of the neural networks models are severely limited in their abilities to solve real world problems. For problems such as speech recognition, image processing, natural language processing and decision-making, it is not normally possible to see a direct mapping of the given problem on to a neural network model. These are natural tasks, which human beings are good at, but we still do not understand how we do them. Hence it is a challenging task to find suitable neural network models to address these problems.

Automatic recognition, description, classification and grouping of patterns are important problems in a variety of engineering and scientific disciplines such as biology, psychology, medicine, marketing, computer vision, artificial intelligence and remote sensing. In the most pattern recognition problems, patterns have a dynamic nature and non-adaptive algorithms (instruction sets) will fail to give a realistic solution to the problem. So in these cases, adaptive algorithms are used and among them, neural networks have the greatest hit. For example, the defenses applications very frequently need to record, detect, identify and classify images of objects or signals coming from various directions and from various sources- static or dynamic [5]. There are many applications in remote sensing like deforestation, effects of natural and manmade disasters, migration in the path of river due to dynamic nature of earth plates where study of dynamic data is needed.

Artificial Neural Networks (ANN) can play a role in such applications because of their capability to model nonlinear 
processes and to identify unknown patterns and images based on their learning model, or to forecast certain outcomes by extrapolation. On the basis of properties like steepness of slopes, local relief (the maximum local difference in elevation) and cross sectional forms of valley and divides, and texture of the surface material etc. considerable foresight can be achieved regarding temporal changes in land patterns. The land pattern of landform differences is strongly related in the arrangement of such other features of the natural environment as climate, soils, and vegetations.

In the present work we wish to classify satellite images using ANN's pattern recognition and classification capabilities. The Unsupervised Classification approach uses self organizing feature map to classify the patterns. The Selforganizing feature maps (SOFM) transform the input of arbitrary dimension into a one or two dimensional discrete map subject to a topological (neighborhood preserving) constraint.

The feature maps are computed using Kohonen unsupervised learning [6]. The output of the SOFM can be used as input to a supervised classification neural network such as the MLP. This network's key advantage is the clustering produced by the SOFM which reduces the input space into representative features using a self-organizing process. Hence the underlying structure of the input space is kept, while the dimensionality of the space is reduced.

\section{ARTIFICIAL NEURAL NETWORK}

The ANN is usually implemented using electronic components (digital or analog) and/or simulated on a digital computer. It employs massive interconnection of simple computing cells called 'neurons' or 'processing elements (PE)". It resembles the brain in two ways: Knowledge is acquired by the network through learning process and Inter neuron connection strengths (synaptic weights) are responsible for storing the knowledge. The way the synaptic weights change is what makes the design of ANNs. An ANN works as follows:

A neuron receives inputs from a large number of other neurons or from an external stimulus. Weighted sums of these inputs are fed into a nonlinear activation function. The output of this function is fanned out (distributed) to connections to other neurons. The topology of neuron connections defines the flow of information in the network. The way the weights are adjusted in the network constitutes the learning process. Thus the three essential components of an ANN computational system are- activation function, architecture, and, the learning law.

Due to the differences in these three components, different ANN structures are explored for various applications and these structures differ in their computational complexities and requirements. The main attributes of neural processing are its nonlinear and adaptive learning capability, which enable machines to recognize possible variations of a same object or pattern and/or to identify unknown functions and mappings based on a finite set of training data, which can be noisy with missing information. Based on this 'Training by example' property with strong support of statistical and optimization theories, neural networks are becoming one of the most powerful and appealing nonlinear and adaptive data analysis tools for a variety of signal processing applications [7].

\section{ClassificAtion OF SATELLITE IMAGES USING SOFM}

In this work self-organizing feature map network identifies a winning neuron using the same procedure as employed by a competitive layer. However, instead of updating only the winning neuron, all neurons within a certain neighborhood of the winning neuron are updated using the Kohonen rule.

The weights of the winning neuron (a row of the input weight matrix) are adjusted with the Kohonen learning rule. Supposing that the $i^{\text {th }}$ neuron wins, the elements of the $i^{\text {th }}$ row of the input weight matrix are adjusted as shown below [8].

$$
\mathrm{iIW} 1,1(\mathrm{q})=\mathrm{iIW} 1,1(\mathrm{q}-1)+\alpha(\mathrm{p}(\mathrm{q})-\mathrm{iIW} 1,1(\mathrm{q}-1))
$$

The Kohonen rule [13] allows the weights of a neuron to learn an input vector, and because of this it is useful in recognition applications. Thus, the neuron whose weight vector was closest to the input vector is updated to be even closer. The result is that the winning neuron is more likely to win the competition the next time a similar vector is presented and less likely to win when a very different input vector is presented.

As more and more inputs are presented, each neuron in the layer closest to a group of input vectors soon adjusts its weight vector toward those input vectors. Eventually, if there are enough neurons, every cluster of similar input vectors will have a neuron that outputs 1 when a vector in the cluster is presented, while outputting a 0 at all other times. Thus, the competitive network learns to categorize the input vectors it sees [9].

Finding the negative distance between input vector $\mathrm{p}$ and the weight vectors and adding the biases $b$ compute the net input. If all biases are zero, the maximum net input a neuron can have is 0 . This occurs when the input vector $\mathrm{p}$ equals that neuron's weight vector. After this computation all neurons within a certain neighborhood $\mathrm{Ni}^{*}(\mathrm{~d})$ (2) of the winning neuron are updated using the Kohonen rule. Specifically, we adjust all such neurons as follows.

$$
\begin{gathered}
\operatorname{iIW} 1,1(q)=\operatorname{iIW} 1,1(q-1)+\alpha(p(q)-i I W 1,1(q-1)) \\
\text { or } \\
\operatorname{iw}(q)=(1-\alpha) \operatorname{iw}(q-1)+\alpha p(q)
\end{gathered}
$$

Here the neighborhood $\mathrm{Ni}^{*}(\mathrm{~d})$ (2) contains the indices for all of the neurons that lie within a radius " $\mathrm{d}$ " of the winning neuron $i^{*}(1)$.

$$
\mathrm{Ni}(\mathrm{d})=\{\mathrm{j}, \mathrm{dij} \leq \mathrm{d}\}
$$

Thus, when a vector is presented the weights of the winning neuron and its close neighbors move toward. Consequently, after many presentations, neighboring neurons will have learned vectors similar to each other [10]. 


\section{EXPERIMENTAL RESULT}

A SOFM classification is experimented on a IRS/1D L3 Image over a specific area for a specific period. This image of size $1200 * 900$ consists of 4 bands 0.52 - 059 microns (B2), 0.62 - 0.68 microns (B3), $0.77-0.86$ microns (B4) and 1.55 1.7 microns (B5). The aim of the classification is to distinguish between dense vegetation, coarse vegetation, water body and urban area [11]. The important requirement of the classification is the Features of the Image. Features are the characteristics of the images quantified e.g. statistical features such as mean, minimum, maximum, variance, covariance, correlation and standard deviation [12]. Other examples of the features are band ratio and difference between two images of the same area (i.e. taken at difference of time span)

Here we have shown the classified image with the various colors coding having unique color using MATLAB [14].

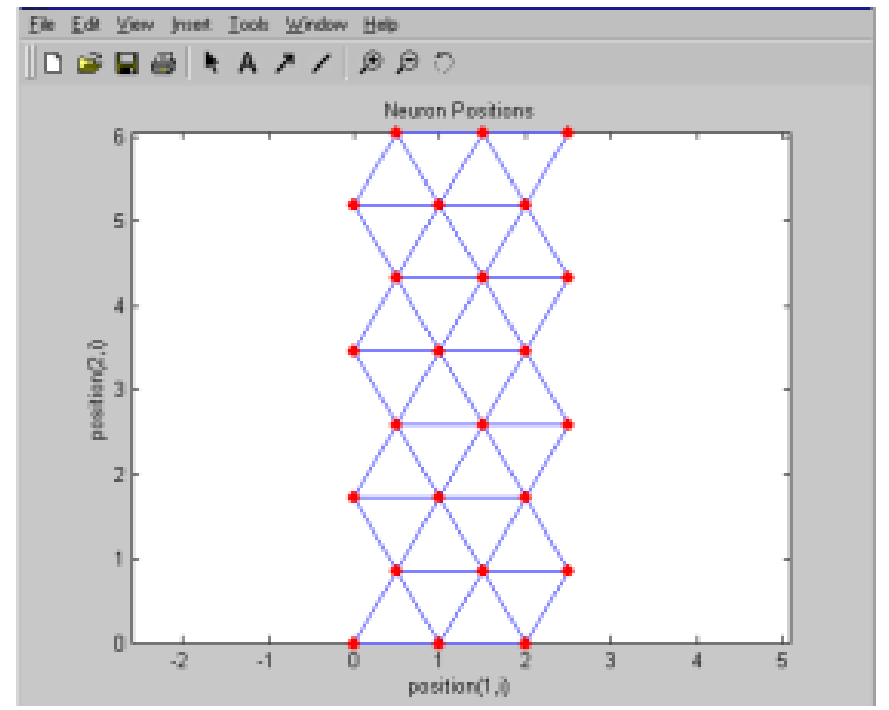

Fig.1. Neuron Positions

\section{CONCLUSION}

In the present work the classifications for multispectral satellite images using self organizing feature map have been done. The classification difference taken over a period of time can be used for trend analysis using SOFM. Such techniques can indeed be applied for a variety of purposes such as deforestation, archeology, urban planning and development, damage assessment, defense intelligence, and environmental monitoring, weather forecasting etc.

The work is executed using the Image Processing and Neural Network toolboxes of MATLAB because of the definite advantage of flexibility and expandability $[12,15]$.

\section{REFERENCES}

[1] S. Kauffman, "Origins of Order", Oxford University Press, 1993.

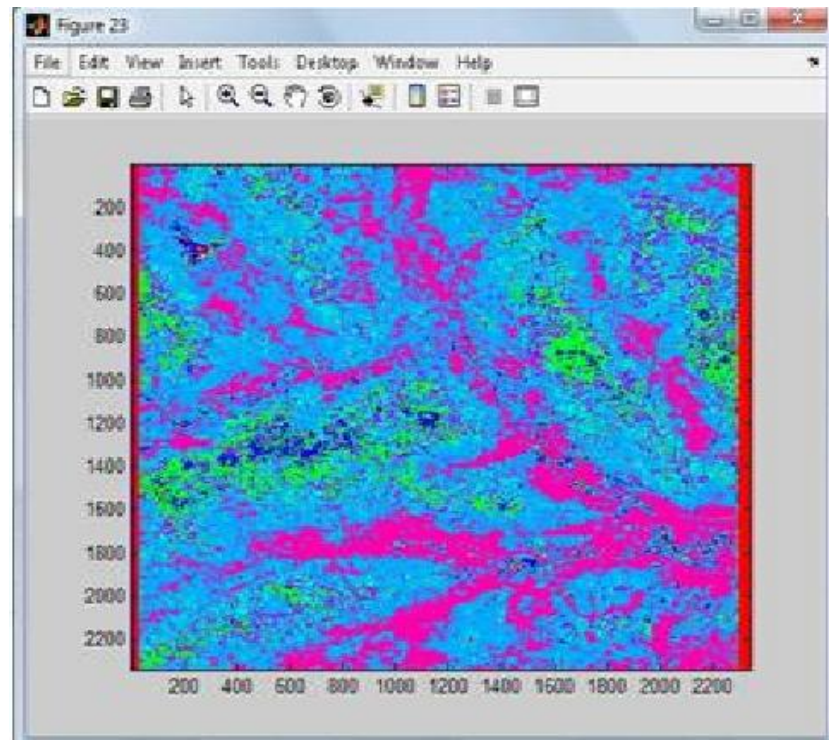

Fig.2. Classified Image

[2] B.Yam, "Dynamics of Complex Systems", Addison-Wesley, 1997.

[3] R. Rojas, "Neural Networks: A Systematic Introduction". Springer, Berlin, 1996.

[4] D. Rumelhart and J. McClelland, "Parallel Distributed Processing", MIT Press, Cambridge, 1986

[5] A. Mangal, P. Mathur and R. Govil. "Trend Analysis in satellite Imagery Using SOFM". Apaji Institute of Mathematics \& Computer Technology, Banasthali Vidhyapith, Rajasthan, India.

[6] A.S.Weigend and N.Gershenfeld, editors.Time series prediction: "forecasting the future and understanding the past", Wesley, 1993.

[7] P. Sharma and U. Mutreja, "Analysis of Satellite Images using Artificial Neural Network", International Journal of Soft Computing and Engineering (IJSCE) ISSN: 2231-2307, Volume-2, Issue-6, January 2013.

[8] G. A. Carpenter, "The ART of adaptive pattern recognizing neural Network”, IEEE Comput. Mag., pp. 77-88, Mar. 1988.

[9] A. B. Yegnarayana, "Artificial Neural Networks", Prentice Hall of India Pvt. Ltd, New Delhi, 1999.

[10] R. C. Gonzalez, "Richard E Woods Digital Image Processing", Pearson Education, Inc Second Edition.

[11] H. S. Chae, S. J. Kim and J. A, Rye, "A Classification of Multi Spectral Landsat TM Data using Principal Component Analysis and ANN", IEEE 1997.

[12] D. Singh, G. Sharma and G. Bhardwaj, "Application of Artificial Neural Network to Satellite Images Classification", Academia Arena, vo. 4 issue 4, 2012.

[13] N. R. Euliano and J. C. Principe. "Self- Spatio Temporal Self Organising Feature Map", Computational NeuroEngineering Laboratory, Department of Electrical Engineering, University of Florida, Gainesville, FL 32611.

[14] T.Koskela, M. Varsta, J.Heikkonen, and K. Kaski. "Time series prediction using recurrent SOM with local linear models. Int. J. of knowledge-Based Intelligent Engineering System”, Volume 2, Issue 1, pp 60-68,1998.

[15] G. J. Chappell and J. G. Taylor, "The temporal Kohonen map, Neural Networks", Volume 6 Issue 3, pp 441-445, 1993. 